\title{
Prevalence and Clinical Features of Seizure Attacks in Psychiatric Wards: A Local
}

\section{Review}

Saeed Shoja Shafti ${ }^{*}$, Alireza Memarie, Masomeh Rezaie, Masomeh Hamidi

University of Social welfare and Rehabilitation sciences, Razi Psychiatric Hospital, Tehran, Iran

*Corresponding Author: Saeed Shoja Shafti, Professor of Psychiatry, University of Social welfare and Rehabilitation sciences, Razi Psychiatric Hospital, Tehran, Iran. E-mail: ssshafti@gmail.com

Received date: September 18, 2019; Accepted date: October 11, 2019; Published date: October 15, 2019

Citation : Shoja Shafti S., Memarie A., Rezaie M., Hamidi M. (2019) Prevalence and Clinical Features of Seizure Attacks in Psychiatric Wards: A Local Review, J Neuroscience and Neurological Surgery. 5(1): Doi: 10.31579/2578-8868 /093

Copyright : (c) 2019 Saeed Shoja Shafti. This is an open-access article distributed under the terms of The Creative Commons Attribution License, which permits unrestricted use, distribution, and reproduction in any medium, provided the original author and source are credited.

Abstract:
Introduction: Behavioral and cognitive dysfunction is frequently observed in patients with recurrent seizures and represents an
important challenge in treating these patients. In the present assessment the incidence and clinical profile of seizure attacks have
been probed among a great sample of non-western psychiatric inpatients and compared with the available data in literature
regarding prevalence and other associated clinical physiognomies.
Methods: All inpatients that had suffered seizure attack during the last sixty-four months had been included in the present
study. Clinical diagnosis, as well, was in essence based on Diagnostic and Statistical Manual of Mental Disorders, 5th edition
(DSM-5).
Results: While epilepsy had been identified as the primary cause of seizure ( $82.92 \%), 17.64 \%$ of them had experienced their
first attacks during hospitalization. Annual incidence of seizure in psychiatric inpatients, on the whole, was around $0.07 \%$. It
was almost 0.06 in identified epileptic cases and about $0.01 \%$ with respect to the first time unprovoked epileptic attack. Also,
the seizure attacks were significantly more prevalent among male psychiatric inpatients than female patients. Schizophrenia and
bipolar disorder were significantly more prevalent than substance abuse, schizoaffective and depression, as comorbid
psychiatric disorders among patients who had suffered seizure attacks, generally, and known cases of epilepsy, particularly.
Conclusion: While, etiologically, epilepsy had been identified as the primary cause of seizure in psychiatric inpatients,
schizophrenia and bipolar disorder were significantly more prevalent, as comorbid psychiatric disorders, in comparison with
other primary psychiatric illnesses. Male gender, as well, could be considered as a risk factor.
Key words: seizure; epilepsy; schizophrenia; mood disorder; neuropsychiatry.

\section{Introduction}

It is notable that the incidence of unprovoked seizures in the placebo arms of RCTs of antidepressants and antipsychotics is approximately 15 -fold higher, proposing that both depression and psychosis are risk factors for seizures [1]. More recently, a bidirectional link between epilepsy and several psychiatric diseases has been revealed, whereby not only do people with epilepsy have a higher risk of developing a psychiatric illness, but people with psychiatric disorder have a higher risk of developing epilepsy [2,3]. This bidirectional relationship exists for depression, anxiety, psychosis and suicidality [4]. Thus, the occurrence of seizures may, in some cases, be the expression of the natural progression of a psychiatric disorder, unrelated to the use of psychotropics [1]. Alternatively, people with epilepsy have an elevated prevalence of several psychiatric disorders including depression (22.9\%), anxiety $(20.2 \%)$ and psychosis $(5.2 \%)[4,5]$. Mania is considered rare [6]. Suicide is fivefold higher in epileptic patients compared to the general population [7] and is an important cause of premature mortality [8]. The link between epilepsy and mental illness is bidirectional as patients with depression, anxiety and psychosis have an augmented risk of developing new-onset epilepsy [3]. Suicide attempts are also associated with the development of epilepsy [7]. It deserves to be mentioned that while in most developed countries, incidence rates of epilepsy range from 40 to 70 per $100,000(9,10)$, in developing countries, the rates may be as high as 100 to 190 per 100,000 [9]. Also, Men are 1.0 to 2.4 times more likely to have epilepsy than are women [9]. As said before, behavioral and cognitive dysfunction is frequently observed in patients with epilepsy and denotes an important challenge in treating these patients. A complex array of factors influence the neuropsychiatric effect of epilepsy, including the cause of the epilepsy, location of epileptogenic focus, age at onset, nature of the epilepsy syndrome, seizure type, frequency, duration of epilepsy, medications used for treatment, and psychosocial factors. Behavioral disturbances are most common with complex partial seizures and seizures involving foci in the temporolimbic structures [6]. People with epilepsy have nearly 2.5 times the risk of developing schizophrenia and almost 3 times the risk of developing a schizophrenia-like psychosis compared with the general population. The data suggested that the risk also increases with an increasing number of hospital admissions for epilepsy and with people first admitted for epilepsy at later ages. The underlying causal mechanism for the association of epilepsy with schizophrenia or schizophrenia-like psychosis is unknown but may have features in common with postictal psychosis and likely involves bilateral cerebral dysfunction within frontal subcortical circuits and probably, temporal subcortical circuits, as well [6]. In this regard, hippocampus is considered the regional focal point of the brain, playing an important role in cognition, psychosis, and seizure activity and potentially suggesting common etiologies and pathophysiology of temporal lobe epilepsy (TLE) and schizophrenia [11]. On the other hand, the association with epilepsy is not limited to schizophrenia and other psychiatric disorders, such as acute stress disorder, anxiety, depression, bipolar disorder, attention deficit hyperactivity disorder, sleep disorders, and movement disorders, as well, may be involved. Moreover, it is conceivable that persons with schizophrenia are susceptible to epilepsy $[12,13]$. Coincidentally, the onsets of schizophrenia as well as temporal TLE frequently occur around adolescence, the final stage of the brain's development. Perhaps, it would be reasonable to speculate that recurrent and prolonged seizures result in alterations to the developing brain architecture, which serves as a trigger to disassemble the molecules involved in forming improper neural networks. Formation of improper neuronal networks could be deleterious to brain functionality, leading to devastating psychiatric conditions [12]. Though psychoses correlate positively with the multiplicity of seizures and inversely with their frequency, it is the highest in pharmoco-resistant cases. A 
genetic factor, as well, is likely to play a role [14]. While the temporal lobe hypothesis of schizophrenia put forward in the 1960s notes that episodes with paranoid psychoses are more prevalent in TLE, some of the scholars believe that psychosis of epilepsy is a distinct nosologic entity differing from schizophrenia not only in clinical details but also in neurobiological aspects [15], and mood disorder symptoms, such as depression and mania, are seen less often in epilepsy than are schizophrenia-like symptoms [16]. In the present assessment the incidence and clinical profile of seizure attacks have been probed among a great sample of non-western psychiatric inpatients and compared with the available data in literature regarding prevalence and other associated clinical physiognomies. Methods:

Razi psychiatric hospital in south of capital city of Tehran, as one of the largest and oldest public psychiatric hospitals in the Middle East, which has been established formally in 1917 and with a capacity around 1375 active beds, had been selected as the field of study in the present retrospective assessment. For evaluation, all inpatients that had suffered seizure during the last sixty-four months had been included in the present study. Clinical diagnosis, as well, was in essence based on Diagnostic and Statistical Manual of Mental Disorders, 5th edition (DSM-5) [17].

\section{Statistical analysis:}

Analysis of dependent variables had been accomplished by 't-test' and appraisal of independent variables had been explored by means of 'comparison of proportions'. Statistical significance, as well, had been defined as a $p$ value $\leq 0.05$. MedCalc Statistical Software version 15.2 was used as statistical software tool for analysis.

\section{Results:}

As said by results, among 20118 psychiatric patients hospitalized in razi psychiatric hospital, during the last sixty-four months (April of 2014-August 2019), eighty two had experienced at least one seizure attack during their inpatient management. So, while annual incidence of seizure in inpatients, in general, was around $0.07 \%$, it was almost 0.06 in identified epileptic cases and about $0.01 \%$ with respect to the first time unprovoked attack with no detectable medical reason. Also, with respect to gender difference and according to data, while there was no significant difference regarding age between male (mean: $42.74 \pm 12.725 \mathrm{y} / \mathrm{o}$ ) and female (mean: $48.03 \pm 15.34 \mathrm{y} / \mathrm{o}$ ) group of patients $(\mathrm{t}=1.66, \mathrm{p}<0.10$, CI 95\%: $-1.04,11.63)$, in the present estimation the seizure attacks were significantly more prevalent among male psychiatric inpatients than female inpatients $(\mathrm{z}=4.06$, $\mathrm{p}<0.00$, CI 95\%:0.16, 0.47). Concerning etiology, in the current appraisal, epilepsy had been identified as the main cause of seizure attacks (82.92\%) (Table 1). Among them 17.64\% ( $n=12)$ had experienced their first attacks during hospitalization and the remaining had experienced it in the past, in different occasions during the last 12 years (mean: $7.45 \pm 4.37$ years). Two patients had experienced their first attacks shortly after termination of their prescribed electroconvulsive therapy (six-session). Moreover, with respect to prevalence of primary psychiatric disorders among patients, who have experienced seizure attacks during the last 64 months, there was no significant difference, in general, between thought disorders (schizophrenia plus schizoaffective) and mood disorders (bipolar disorder plus major depressive disorder) $(\mathrm{z}=1.75, \mathrm{p}<0.07, \mathrm{CI} 95 \%$ : $0.01,0.28)$, from one hand, and between schizophrenia and bipolar disorder $(\mathrm{z}=1.30, \mathrm{p}<0.19$, CI 95\%: $-0.04,0.24)$, on the other hand. But both schizophrenia and bipolar disorder were significantly more prevalent than substance abuse $(\mathrm{z}=4.72, \mathrm{p}<0.00$, CI 95\%: 0.18, 0.44, and $\mathrm{z}=3.54, \mathrm{p}<0.00$, CI 95\%: 0.09,0.34, respectively), schizoaffective and depression (Table 2). The same pattern was relatively valid as regards the identified epileptic cases (Table 3 ).

\section{Discussion:}

While psychiatric ailments repeatedly occur in patients with epilepsy, the diagnosis is regularly missed and therapeutic opportunities are often lost. These comorbidities assume greater importance as epidemiological data show their frequent relationship with decreased function and quality of life, and advances in neurobiology better define their pathophysiological association and therapies. So, maybe epilepsy presents a model for understanding psychiatric illness. Decoding the role of different biological and environmental risk factors may help identify high-risk patients and allow for early intervention. Antiepileptic drugs are frequently used to manage psychiatric syndromes although the mechanisms of their psychotropic action remain ambiguous. As recognition and management of comorbid psychiatric disorders in epilepsy remain suboptimal, we need to increase the awareness of physicians, patients, their caregivers, and the health care system. Better recognition will help to improve and implement proper diagnostic and treatment plans, and improve functional outcomes and quality of life in people with epilepsy (18). While the main psychiatric complications in epilepsy include depression, anxiety disorders, and psychosis, comorbidity of epilepsy may be due to a shared pathophysiological mechanism. Nonetheless, coincidence or selection bias cannot at all times be excluded. In addition, genetic, psychosocial and iatrogenic factors may also contribute to the comorbidity [19]. On the other hand, TLE and psychosis coexist more commonly than chance would foresee. The discovery of shared mechanisms and/or affected neurochemicals in temporal lobe epilepsy and schizophrenia might disclose important clues on the vulnerability of such patients to psychotic symptoms and be an opportunity for new treatment development [20]. Impairment and deregulation among critical anatomical regions, such as the hippocampus, amygdala, thalamus, and the temporal, frontal and cingulate cortices, might predispose TLE brains to psychosis. Studies of the effects of kindling and injection of neuroactive substances on performance and electrophysiological configurations may offer a model of how limbic seizures in humans raise the vulnerability of epileptic patients to psychiatric illnesses [12]. Back to our study and along with the results, while the annual incidence of seizures, on the whole, in our study was somewhat similar to Sander (9), with respect to its rate in developed countries, and higher than Ngugi AK et al. [10], it was very lower than estimation of Sander [9], with respect to its rate in developing countries. Also, with respect to the first time unprovoked attack our assessment was lower than both of them. With respect to gender difference, as well, our finding was to some degree analogous with the estimates of Sander [9]. Respecting prevalence of psychiatric comorbidities, our conclusions were not in harmony with the findings of Cunningham et al. [6] and Vuilleumier et al. [14], who had found depression as the most prevalent comorbidity and psychosis as the fewest one. On contrary, among our samples, the schizophrenia was the most prevent psychiatric comorbidity, which was significantly more prevalent than depression. Also, bipolar disorder was the second most prevalent psychiatric disorder among our epileptic cases, which was not in accord with the findings of Cunningham et al. [6], who had considered it a rare comorbidity in epileptic patients. Then again, our results were to some extent consistent with the verdicts of Lavery et al. [16], who has indicated that mood disorder symptoms, such as depression and mania, are seen less often in epilepsy than are schizophrenia-like symptoms. Furthermore, in opposite to Scott et al.[4], no remarkable anxiety disorder had been detected in the current survey. In general, patients who have epilepsy or recurrent seizure attacks face many challenges resulting from their ailment and have frequent psychiatric comorbidities. Recognition of these disorders is increasing and is having a positive impact on patients' quality of life. Recent recommendations about a new classification system for psychiatric disorders related specially to epilepsy and based on the relationship of symptoms to seizures, antiepileptic medications, and electroencephalogram changes demand further investigations [21]. Principally insofar psychiatric syndromes specific to epilepsy can be identified, correlation of clinical phenomena with relatively well-understood pathophysiology in epilepsy will permit progresses in the understanding of psychiatric disorder. This progress should move the management of patients who have recurrent seizure attacks toward an allinclusive biopsychosocial model that focuses on the whole person rather than simply on the disease process [21]. Restricted period of study, due to inadequate registration and documents in the last decades, and thus insufficient number of cases who had suffered seizure attacks, as accessible samples, in 
spite of greatness of the field of study, do not permit generalization of outcomes to more than a preliminary survey. No doubt, further methodical studies in future will improve our clinical idea concerning diagnosis and management of this vital problem.

\section{Conclusion:}

While, etiologically, epilepsy had been identified as the primary cause of seizure in psychiatric inpatients, schizophrenia and bipolar disorder were significantly more prevalent, as comorbid psychiatric disorders, in comparison with other primary psychiatric illnesses. Male gender, as well, could be considered as a risk factor

\begin{tabular}{|c|c|c|}
\hline Identified causes & Number & \% \\
\hline Epilepsy & 68 & 82.92 \\
\hline Encephalitis & 1 & 1.21 \\
\hline Meningitis & 2 & 2.43 \\
\hline Hypertensive encephalopathy & 1 & 1.21 \\
\hline Substance induced & 2 & 2.43 \\
\hline Lithium toxicity & 1 & 1.21 \\
\hline Heat trauma & 3 & 3.65 \\
\hline Hyponatremia & 3 & 3.65 \\
\hline Hypoglycemia & 1 & 1.21 \\
\hline
\end{tabular}

Table 1: Etiology of seizure attacks.

\begin{tabular}{|c|c|c|}
\hline Psychiatric disorder & Number & \% \\
\hline & & \\
\hline Schizophrenia & 36 & 43.90 \\
\hline Bipolar disorder & 25 & 30.48 \\
\hline schizoaffective & 5 & 6.09 \\
\hline Substance abuse & 7 & 8.53 \\
\hline Depression & 2 & 2.43 \\
\hline
\end{tabular}

Table 2: Comorbid psychiatric disorders among patients with seizure attacks.

\begin{tabular}{|c|c|c|}
\hline Psychiatric disorder & Number & \% \\
\hline Schizophrenia & 26 & 38.23 \\
\hline Bipolar disorder & 22 & 32.35 \\
\hline schizoaffective & 4 & 5.88 \\
\hline Substance abuse & 7 & 10.28 \\
\hline Depression & 2 & 2.94 \\
\hline
\end{tabular}

Table 3: Comorbid psychiatric disorders among identified epileptic patients.

\section{References:}

1. Alper $\mathrm{K}$ et al. Seizure incidence in psychopharmacological clinical trials: an analysis of Food and Drug Administration (FDA) summary basis of approval reports. Biol Psychiatry 2007; 62:345-354.

2. Kanner AM. Management of psychiatric and neurological comorbidities in epilepsy. Nat Rev Neurol 2016; 12:106-116.

3. Hesdorffer DC. Comorbidity between neurological illness and psychiatric disorders. CNS Spectr 2016; 21:230-238

4. Scott AJ et al. Anxiety and depressive disorders in people with epilepsy: a meta-analysis. Epilepsia 2017; 58:973-982.
5. Clancy MJ et al. The prevalence of psychosis in epilepsy; a systematic review and meta-analysis. BMC Psychiatry 2014; $14: 75$.

6. Cunningham M G, Goldstein M, Katz D, et al. Coalescence of psychiatry, neurology, and neuropsychology: from theory to practice, Harv Rev Psychiatry 2006; 14:127-140

7. Hesdorffer DC et al. Occurrence and recurrence of attempted suicide among people with epilepsy. JAMA Psychiatry 2016; 73:80-86.

8. Thurman DJ et al. The burden of premature mortality of epilepsy 
in high-income countries: a systematic review from the Mortality Task Force of the International League Against Epilepsy. Epilepsia 2017; 58:17-26.

9. Sander, J. W. 2003, The epidemiology of epilepsy revisited, Curr Opin Neural, 2003; 16: 165-170.

10. Ngugi AK et al. Incidence of epilepsy: a systematic review and meta-analysis. Neurology 2011; 77:1005-1012.

11. Nakahara S, Adachi M, Ito H, Matsumoto M, Tajinda K, Erp TGM. Hippocampal Pathophysiology: Commonality Shared by Temporal Lobe Epilepsy and Psychiatric Disorders. Neuroscience Journal 2018:1-9.

12. Kandratavicius L, Lopes-Aguiar C, Bueno-Júnior LS, RomcyPereira RN, Hallak JE, Leite JP. Psychiatric comorbidities in temporal lobe epilepsy: possible relationships between psychotic disorders and involvement of limbic circuits. Braz J Psychiatry 2012;34(4):454-66.

13. B. Ettinger, M. L. Reed, J. F. Goldberg, and R. M. A. Hirschfeld, "Prevalence of bipolar symptoms in epilepsy vs other chronic health disorders," Neurology 2005; 65 (4): 535-540, 2005.

14. Vuilleumier P, Jallon P. [Epilepsy and psychiatric disorders: epidemiological data]. Rev Neurol (Paris). 1998;154(4):305-17.

15. Tebartz Van Elst L, Baeumer D, Lemieux L, Woermann FG, Koepp M, Krishnamoorthy S, Thompson PJ, Ebert D, Trimble MR. Amygdala pathology in psychosis of epilepsy: A magnetic resonance imaging study in patients with temporal lobe epilepsy. Brain 2002;125(Pt 1):140-9.
16. Lavery LL, Whyte EM. Other cognitive and mental disorders due to a general medical condition. In: Sadock BJ, Sadock VA, Ruiz P, eds. Kaplan \& Sadock's Comprehensive Textbook of Psychiatry. 9th ed. Philadelphia: Lippincott Williams \& Wilkins; 2009:1207.

17. American Psychiatric Association (APA). Diagnostic and Statistical Manual of Mental Disorders. 5th ed. Washington, DC: American Psychiatric Association, 2013.

18. Devinsky O.Psychiatric comorbidity in patients with epilepsy: implications for diagnosis and treatment. Epilepsy Behav 2003;4 (4): 2-10.

19. Beyenburg S, Damsa C. [Psychiatric comorbidity in epilepsy]. Bull Soc Sci Med Grand Duche Luxemb 2005;(3):283-92.

20. Kandratavicius L, Hallak JE Leite JP. What are the similarities and differences between schizophrenia and schizophrenia-like psychosis of epilepsy? A neuropathological approach to the understanding of schizophrenia spectrum and epilepsy. Epilepsy Behav 2014;38:143-7.

21. Kandratavicius L, Lopes-Aguiar C, Bueno-Júnior LS, RomcyPereira RN, Hallak JE, Leite JP. Psychiatric comorbidities in temporal lobe epilepsy: possible relationships between psychotic disorders and involvement of limbic circuits. Braz J Psychiatry 2012;34(4):454-66.

22. Marcangelo MJ, Ovsiew F. Psychiatric aspects of epilepsy. Psychiatr Clin North Am 2007;30(4):781-802 